\title{
Finiteness results for Diophantine triples with repdigit values
}

by

Attila Bérczes (Debrecen), Florian Luca (Johannesburg), István Pink (Debrecen and Salzburg) and Volker Ziegler (Salzburg)

1. Introduction. A classical Diophantine $m$-tuple is a set of $m$ positive integers, $\left\{a_{1}, \ldots, a_{m}\right\}$, such that $a_{i} a_{j}+1$ is a square for all $1 \leq i<j \leq m$. Dujella [6] proved that there is no Diophantine sextuple and that there are only finitely many Diophantine quintuples. A folklore conjecture is that there are no Diophantine quintuples. Various variants of the notion of Diophantine tuples have been considered in which the set of squares has been replaced by some other arithmetically interesting subsets of the positive integers. For instance, the case of $k$ th powers was considered in [3], while the case of the members of a fixed binary recurrence was considered in [7, 13, 14]. In [10], it is proved that there is no triple $\{a, b, c\}$ of positive integers such that all of $a b+1, a c+1, b c+1$ belong to the sequence $\left\{u_{n}\right\}_{n \geq 0}$ of the recurrence $u_{n}=A u_{n-1}-u_{n-2}$ for $n \geq 2$ with initial values $u_{0}=0$ and $u_{1}=1$. For related results, see [1, 8, 9]. Diophantine tuples with values in the set of $S$-units for a fixed finite set $S$ of primes were considered in [16, 19]. For a survey on this topic, we recommend the Diophantine $m$-tuples page maintained by A. Dujella [5].

Here we take an integer $g \geq 2$ and recall that a repdigit $N$ in base $g$ is a positive integer all of whose base $g$ digits are the same. That is,

$$
N=d \frac{g^{k}-1}{g-1} \quad \text { for some } d \in\{1, \ldots, g-1\} .
$$

These numbers fascinated both mathematicians and amateurs. Questions concerning Diophantine equations involving repdigits have been considered by Keith [11], Marques and Togbé [17] and Kovács et al. [12], to name just

2010 Mathematics Subject Classification: Primary 11D61.

Key words and phrases: Diophantine sets, repdigit numbers.

Received 19 February 2015; revised 28 August 2015.

Published online 23 December 2015. 
a few. In this paper, we combine the Diophantine tuples with repdigits and thus consider Diophantine triples having products increased by 1 in the set of repdigits in a fixed base $g$.

To avoid trivialities, we only look at repdigits with at least two digits. That is, the parameter $k$ appearing in (1.1) satisfies $k \geq 2$. We denote by $\mathcal{R}_{g}$ the set of all positive integers that are repdigits in base $g$. In this paper, we are interested in triples $(a, b, c) \in \mathbb{N}^{3}$ with $c<b<a$ such that $a b+1, a c+1$ and $b c+1$ are all elements of $\mathcal{R}_{g}$. Let us denote by $\mathcal{D}_{g}$ the set of all such triples. The reason why we exclude the one-digit numbers from our analysis is, that in some sense, these are degenerate examples. Furthermore, if we allow $a b+1, a c+1$ and $b c+1$ to be one-digit numbers in a large base $g$, we will have many small examples, which however are of no interest.

Our main result is the following.

Theorem 1.1. Assume that $(a, b, c) \in \mathcal{D}_{g}$. Then

$$
\begin{array}{ll}
a \leq \frac{g^{186}-2}{2} & \text { for all integers } g \geq 2, \text { and } \\
a \leq \frac{g^{124}-2}{2} & \text { for all integers } g \geq 10^{6} .
\end{array}
$$

Moreover,

$$
\# \mathcal{D}_{g} \leq \frac{(185 g-185)(185 g-186)(185 g-187)}{6}
$$

for all bases $g$ and

$$
\# \mathcal{D}_{g} \ll g^{1+o(1)} \quad \text { as } g \rightarrow \infty .
$$

In the next section, we estimate the greatest common divisor of two numbers of a special shape, which is an important step in the proof of Theorem 1.1. In Section 3, we prove Theorem 1.1 except for the asymptotic bound 1.2 , which is proved later in Section 4 .

We want to emphasize that our proof of Theorem 1.1 yields a rather efficient algorithm to compute $\mathcal{D}_{g}$ for a given $g$. In particular, we have computed all sets $\mathcal{D}_{g}$ for $2 \leq g \leq 200$, and we give the details and the results of this computation in the last section.

2. Estimates for the GCD of some numbers of special shape. The main result of this section is:

Lemma 2.1. Let $g \geq 2, k_{1}, k_{2} \geq 1, t_{1}, w_{1}, t_{2}, w_{2}$ be non-zero integers, and set $C:=\max \left\{g,\left|t_{1}\right|,\left|w_{1}\right|,\left|t_{2}\right|,\left|w_{2}\right|\right\}$. Let

$$
\Delta=\operatorname{gcd}\left(t_{1} g^{k_{1}}-w_{1}, t_{2} g^{k_{2}}-w_{2}\right)
$$


and let $X$ be any real number with $X \geq \max \left\{k_{1}, k_{2}, 3\right\}$. If $t_{1} g^{k_{1}} / w_{1}$ and $t_{2} g^{k_{2}} / w_{2}$ are multiplicatively independent, then

$$
\Delta \leq 2 C^{2+5 \sqrt{X}} .
$$

The proof of this lemma depends, among other things, on the following result whose proof is based on the pigeon-hole principle and appears explicitly in [15].

Lemma 2.2 ([15, Claim 1]). Let $m, n$ and $X$ be non-negative integers such that $m$ and $n$ are not both zero, and $X \geq \max \{3, m, n\}$. Then there exist integers $(u, v) \neq(0,0)$ such that

$$
\max \{|u|,|v|\} \leq \sqrt{X} \quad \text { and } \quad 0 \leq m u+n v \leq 2 \sqrt{X} .
$$

Proof of Lemma 2.1. Set $\lambda_{i}=\operatorname{gcd}\left(t_{i} g^{k_{i}}, w_{i}\right)$ for $i=1,2$. We have

$$
t_{i} g^{k_{i}}-w_{i}=\lambda_{i}\left(t_{i} g^{k_{i}} / \lambda_{i}-w_{i} / \lambda_{i}\right) \quad(i=1,2) .
$$

Then $\Delta=\lambda_{1} \lambda_{2} \Delta_{1}$, with

$$
\Delta_{1}=\operatorname{gcd}\left(t_{1} g^{k_{1}} / \lambda_{1}-w_{1} / \lambda_{1}, t_{2} g^{k_{2}} / \lambda_{2}-w_{2} / \lambda_{2}\right) .
$$

Since $\left|\lambda_{i}\right| \leq\left|w_{i}\right| \leq C$ for $i=1,2$, we get the upper bound

$$
\Delta \leq C^{2} \Delta_{1}
$$

Thus, it remains to bound $\Delta_{1}$.

Now, consider the pair of congruences

$$
t_{i} g^{k_{i}} / \lambda_{i} \equiv w_{i} / \lambda_{i}\left(\bmod \Delta_{1}\right) \quad(i=1,2)
$$

and note that $w_{i} / \lambda_{i}$ and $t_{i} g^{k_{i}} / \lambda_{i}$ are invertible modulo $\Delta_{1}$. Indeed, by 2.2 there exists an integer $q$ such that

$$
t_{i} g^{k_{i}} / \lambda_{i}-w_{i} / \lambda_{i}=q \Delta_{1} .
$$

If $w_{i} / \lambda_{i}$ and $\Delta_{1}$ have a common prime factor $p$, then $p \mid t_{i} g^{k_{i}} / \lambda_{i}$, contradicting the fact that $t_{i} g^{k_{i}} / \lambda_{i}$ and $w_{i} / \lambda_{i}$ are coprime. that

By Lemma 2.2, we can find a pair of integers $\left(u_{1}, u_{2}\right) \neq(0,0)$ such

$$
\max \left\{\left|u_{1}\right|,\left|u_{2}\right|\right\} \leq \sqrt{X} \text { and } 0 \leq u_{1} k_{1}+u_{2} k_{2} \leq 2 \sqrt{X} .
$$

Since both sides of 2.2 are invertible modulo $\Delta_{1}$, it makes sense to take the $u_{i}$ th powers on both sides of $(2.2)$ for $i=1,2$. Multiplying the resulting two congruences, we get

$$
\frac{t_{1}^{u_{1}} t_{2}^{u_{2}} g^{k_{1} u_{1}+k_{2} u_{2}}}{\lambda_{1}^{u_{1}} \lambda_{2}^{u_{2}}}-\frac{w_{1}^{u_{1}} w_{2}^{u_{2}}}{\lambda_{1}^{u_{1}} \lambda_{2}^{u_{2}}} \equiv 0\left(\bmod \Delta_{1}\right)
$$

The rational number on the left-hand side of (2.3) is non-zero, since other- 
wise

$$
\left(\frac{t_{1} g^{k_{1}}}{w_{1}}\right)^{u_{1}}\left(\frac{t_{2} g^{k_{2}}}{w_{2}}\right)^{u_{2}}=1,
$$

which implies that $t_{1} g^{k_{1}} / w_{1}$ and $t_{2} g^{k_{2}} / w_{2}$ are multiplicatively dependent because $\left(u_{1}, u_{2}\right) \neq(0,0)$. But this is excluded by our hypothesis. Thus, the left-hand side of $(2.3)$ is a non-zero rational number whose numerator is divisible by $\Delta_{1}$.

Therefore we can write

$$
\frac{t_{1}^{u_{1}} t_{2}^{u_{2}} g^{k_{1} u_{1}+k_{2} u_{2}}}{\lambda_{1}^{u_{1}} \lambda_{2}^{u_{2}}}=\frac{A B_{1} B_{2}}{C_{1} C_{2}}
$$

where $A=g^{k_{1} u_{1}+k_{2} u_{2}}$ and $\left\{B_{1}, B_{2}, C_{1}, C_{2}\right\}=\left\{t_{1}^{\left|u_{1}\right|}, t_{2}^{\left|u_{2}\right|}, \lambda_{1}^{\left|u_{1}\right|}, \lambda_{2}^{\left|u_{2}\right|}\right\}$. Similarly, we have

$$
\frac{w_{1}^{u_{1}} w_{2}^{u_{2}}}{\lambda_{1}^{u_{1}} \lambda_{2}^{u_{2}}}=\frac{D_{1} D_{2}}{E_{1} E_{2}}
$$

where $\left\{D_{1}, D_{2}, E_{1}, E_{2}\right\}=\left\{w_{1}^{\left|u_{1}\right|}, w_{2}^{\left|u_{2}\right|}, \lambda_{1}^{\left|u_{1}\right|}, \lambda_{2}^{\left|u_{2}\right|}\right\}$. Clearly, $|A| \leq C^{2 \sqrt{X}}$, whereas

$$
\max _{i=1,2}\left\{\left|B_{i}\right|,\left|C_{i}\right|,\left|D_{i}\right|,\left|E_{i}\right|\right\} \leq C^{\sqrt{X}} .
$$

First, assume that $u_{1} u_{2} \geq 0$. Then $u_{1}$ and $u_{2}$ have the same sign and

$$
\max \left\{k_{1}, k_{2}\right\}<k_{1}\left|u_{1}\right|+k_{2}\left|u_{2}\right|=\left|k_{1} u_{1}+k_{2} u_{2}\right| \leq 2 \sqrt{X},
$$

which yields

$$
\Delta_{1} \leq \max \left\{\left|t_{1} g^{k_{1}}-w_{1}\right|,\left|t_{2} g^{k_{2}}-w_{2}\right|\right\} \leq 2 C^{1+2 \sqrt{X}} \leq 2 C^{5 \sqrt{X}} .
$$

Next, assume that $u_{1} u_{2}<0$, which immediately implies that $\left\{C_{1}, C_{2}\right\}$ and $\left\{E_{1}, E_{2}\right\}$ have a common element. Without loss of generality, we may assume that $u_{1}>0$ and $u_{2}<0$. Then we can choose $\lambda_{1}^{u_{1}}=C_{1}=E_{1}$ and $\Delta_{1}$ divides the numerator of

$$
\frac{A B_{1} B_{2}}{C_{1} C_{2}}-\frac{D_{1} D_{2}}{C_{1} E_{2}}=\frac{A B_{1} B_{2} E_{2}-C_{2} D_{1} D_{2}}{C_{1} C_{2} E_{2}} .
$$

That is, $\Delta_{1} \mid A B_{1} B_{2} E_{2}-D_{1} D_{2} C_{2}$. Since $A B_{1} B_{2} E_{2}-D_{1} D_{2} C_{2} \neq 0$, we obtain

$$
\Delta_{1} \leq 2 C^{5 \sqrt{X}} .
$$

Therefore, we conclude by (2.5) and (2.6), together with (2.1), that

$$
\Delta \leq 2 C^{2+5 \sqrt{X}} .
$$


3. Proof of Theorem 1.1. Assume that $(a, b, c) \in \mathcal{D}_{g}$. By the definition of $\mathcal{D}_{g}$, we have

$$
\begin{aligned}
& a b+1=d_{3} \frac{g^{n_{3}}-1}{g-1}, \\
& a c+1=d_{2} \frac{g^{n_{2}}-1}{g-1}, \\
& b c+1=d_{1} \frac{g^{n_{1}}-1}{g-1},
\end{aligned}
$$

where $d_{i} \in\{1, \ldots, g-1\}$ and $n_{i} \geq 2$ for $i=1,2,3$. It is clear that $n_{1} \leq n_{2} \leq n_{3}$. Further, we may assume that $g \geq 3$, since if $g=2$, then $d_{1}=d_{2}=d_{3}=1$,

$$
\begin{aligned}
& a b=2^{n_{3}}-2=2\left(2^{n_{3}-1}-1\right), \\
& a c=2^{n_{2}}-2=2\left(2^{n_{2}-1}-1\right), \\
& b c=2^{n_{1}}-2=2\left(2^{n_{1}-1}-1\right),
\end{aligned}
$$

and by multiplying the above equations we get

$$
(a b c)^{2}=8\left(2^{n_{3}-1}-1\right)\left(2^{n_{2}-1}-1\right)\left(2^{n_{1}-1}-1\right),
$$

which yields a contradiction since the left-hand side is a square and the right-hand side is divisible by 8 but not by 16 .

Next, we claim that

$$
n_{3} \leq 2 n_{2} .
$$

In order to prove $(3.2)$, we note that

$$
a<a c+1 \leq g^{n_{2}}-1,
$$

and therefore

$$
g^{n_{3}-1}+g^{n_{3}-2}+\cdots+1 \leq \frac{d_{3}\left(g^{n_{3}}-1\right)}{g-1}=a b+1<a^{2}<\left(g^{n_{2}}-1\right)^{2}<g^{2 n_{2}} .
$$

Thus, $n_{3}<2 n_{2}+1$, and $(3.2)$ is proved. Furthermore, note that

$$
a>(a b+1)^{1 / 2} \geq\left(g^{n_{3}-1}+g^{n_{3}-2}+\cdots+1\right)^{1 / 2}>g^{\left(n_{3}-1\right) / 2} .
$$

Let us fix some notation for the rest of this section. We rewrite (3.1) as:

$$
\begin{aligned}
& a b=\frac{\lambda_{3}}{g-1}\left(\frac{d_{3} g^{n_{3}}}{\lambda_{3}}-\frac{d_{3}+g-1}{\lambda_{3}}\right)=: \frac{\lambda_{3}}{g-1}\left(x_{3}-y_{3}\right), \\
& a c=\frac{\lambda_{2}}{g-1}\left(\frac{d_{2} g^{n_{2}}}{\lambda_{2}}-\frac{d_{2}+g-1}{\lambda_{2}}\right)=: \frac{\lambda_{2}}{g-1}\left(x_{2}-y_{2}\right), \\
& b c=\frac{\lambda_{1}}{g-1}\left(\frac{d_{1} g^{n_{1}}}{\lambda_{1}}-\frac{d_{1}+g-1}{\lambda_{1}}\right)=: \frac{\lambda_{1}}{g-1}\left(x_{1}-y_{1}\right),
\end{aligned}
$$

where

$$
\lambda_{i}=\operatorname{gcd}\left(d_{i} g^{n_{i}}, d_{i}+g-1\right), \quad x_{i}=\frac{d_{i} g^{n_{i}}}{\lambda_{i}}, \quad y_{i}=\frac{d_{i}+g-1}{\lambda_{i}} \quad(i=1,2,3) .
$$


Note that $\operatorname{gcd}\left(x_{i}, y_{i}\right)=1$ for $i=1,2,3$. Hence, the fractions $x_{i} / y_{i}$ are reduced. Note also that $x_{i}>y_{i}$ for $i=1,2,3$.

In order to prove Theorem 1.1, we consider several cases.

CASE 1: $x_{1} / y_{1}$ and $x_{2} / y_{2}$ are multiplicatively dependent and so are $x_{1} / y_{1}$ and $x_{3} / y_{3}$. In this case all the fractions $x_{i} / y_{i}$ with $i=1,2,3$ belong to the same cyclic subgroup of $\mathbb{Q}_{+}^{*}$. Let $\alpha / \beta>1$ be a generator of this subgroup, where $\alpha, \beta$ are coprime integers. Since $x_{i} / y_{i}>1$ for $i=1,2,3$, there exist positive integers $r_{i}$ for $i=1,2,3$ such that

$$
x_{i}=\alpha^{r_{i}} \quad \text { and } \quad y_{i}=\beta^{r_{i}}, \quad i=1,2,3 .
$$

We split this case up into further subcases and start with:

CASE 1.1: There exist $i \neq j$ such that $r_{i}=r_{j}$. Let us start with the case $r_{3}=r_{2}$. We then get

$$
\alpha^{r_{3}}=\frac{d_{3} g^{n_{3}}}{\lambda_{3}}=\frac{d_{2} g^{n_{2}}}{\lambda_{2}}=\alpha^{r_{2}} .
$$

Hence,

$$
g^{n_{3}-n_{2}}=\frac{d_{2} \lambda_{3}}{d_{3} \lambda_{2}} .
$$

We claim that $n_{3}-n_{2} \in\{0,1\}$. Note that $d_{2} \leq g-1$ and $\lambda_{3} \leq 2(g-1)$, which yield $d_{2} \lambda_{3} \leq 2(g-1)^{2}$. In case $d_{3} \lambda_{2} \geq 2$, we obtain

$$
g^{n_{3}-n_{2}} \leq \frac{2(g-1)^{2}}{2}=(g-1)^{2}<g^{2},
$$

so we have $n_{3}-n_{2} \in\{0,1\}$. Therefore, we are left with the case when $d_{3} \lambda_{2}=1$, i.e. $d_{3}=\lambda_{2}=1$. But in this case,

$$
\lambda_{3}=\operatorname{gcd}\left(d_{3} g^{n_{3}}, d_{3}+g-1\right)=\operatorname{gcd}\left(g^{n_{3}}, g\right)=g,
$$

so

$$
g^{n_{3}-n_{2}}=\frac{d_{2} \lambda_{3}}{d_{3} \lambda_{2}}=d_{2} \lambda_{3} \leq g(g-1)<g^{2} .
$$

Thus, in all cases we have $n_{3}-n_{2} \in\{0,1\}$.

Consider now the case $n_{3}-n_{2}=0$. This means that

$$
\frac{d_{3}}{\lambda_{3}}=\frac{d_{2}}{\lambda_{2}} \text {. }
$$

But we also have

$$
\beta^{r_{3}}=\frac{d_{3}+g-1}{\lambda_{3}}=\frac{d_{2}+g-1}{\lambda_{2}}=\beta^{r_{2}} .
$$

Combining (3.5) and (3.6), we obtain $(g-1) / \lambda_{3}=(g-1) / \lambda_{2}$, so $\lambda_{2}=\lambda_{3}$. Now we deduce by $(3.5)$ that $d_{2}=d_{3}$. Altogether this yields $a b+1=a c+1$, contradicting our assumption that $b>c$. 
Now, consider the case $n_{3}-n_{2}=1$. Instead of 3.5 , we now have

$$
\frac{d_{3} g}{\lambda_{3}}=\frac{d_{2}}{\lambda_{2}} .
$$

Combining (3.6) and (3.7), we get

$$
\frac{d_{2}+g-1}{d_{3}+g-1}=\frac{\lambda_{2}}{\lambda_{3}}=\frac{d_{2}}{d_{3} g}
$$

which leads to

$$
d_{3} g\left(d_{2}+g-1\right)=d_{2}\left(d_{3}+g-1\right) .
$$

Assuming that $d_{3} \geq 2$, equation (3.8) yields

$$
2 g^{2} \leq d_{3} g\left(d_{2}+g-1\right)=d_{2}\left(d_{3}+g-1\right) \leq 2(g-1)^{2},
$$

a contradiction, so we may assume that $d_{3}=1$. Inserting $d_{3}=1$ into (3.8) yields

$$
g\left(d_{2}+g-1\right)=d_{2} g,
$$

or equivalently $g(g-1)=0$, which is obviously false. In particular, we have proved that the case $r_{2}=r_{3}$ yields no solution.

The same arguments hold if we replace the quantities $r_{3}, r_{2}, n_{3}, n_{2}, d_{3}, d_{2}$ by $r_{2}, r_{1}, n_{2}, n_{1}, d_{2}, d_{1}$ and $r_{3}, r_{1}, n_{3}, n_{1}, d_{3}, d_{1}$ respectively. Thus, Case 1.1 yields no solution and we assume from now on that $r_{1}, r_{2}$ and $r_{3}$ are pairwise distinct.

CASE 1.2: $r_{3}>\max \left\{r_{1}, r_{2}\right\}$. With our notation, we have

$$
(g-1) a b=\lambda_{3}\left(\alpha^{r_{3}}-\beta^{r_{3}}\right) \quad \text { and } \quad(g-1) a c=\lambda_{2}\left(\alpha^{r_{2}}-\beta^{r_{2}}\right),
$$

and obviously $a(g-1)$ is a common divisor of $\lambda_{3}\left(\alpha^{r_{3}}-\beta^{r_{3}}\right)$ and $\lambda_{2}\left(\alpha^{r_{2}}-\beta^{r_{2}}\right)$. Thus, we have

$$
(g-1) a \mid \operatorname{gcd}\left(\lambda_{3}\left(\alpha^{r_{3}}-\beta^{r_{3}}\right), \lambda_{2}\left(\alpha^{r_{2}}-\beta^{r_{2}}\right)\right) .
$$

Taking a closer look at the greatest common divisor on the right-hand side above, we obtain

$$
(g-1) a \mid \lambda_{2} \lambda_{3}\left(\alpha^{r}-\beta^{r}\right),
$$

where $r=\operatorname{gcd}\left(r_{3}, r_{2}\right)$. Similarly,

$$
(g-1) b \mid \lambda_{3} \lambda_{1}\left(\alpha^{s}-\beta^{s}\right),
$$

where $s=\operatorname{gcd}\left(r_{3}, r_{1}\right)$. Together, the last two inequalities give

$$
(g-1)^{2} a b<\lambda_{1} \lambda_{2} \lambda_{3}^{2} \alpha^{r+s} .
$$

Write $r=r_{3} / \delta$ and $s=r_{3} / \lambda$ for some divisors $\delta>1$ and $\lambda>1$ of $r_{3}$. Note that we cannot have $\delta=\lambda=2$ : this would yield $r_{2}=r_{1}=r_{3} / 2$, which was excluded by Case 1.1. Thus,

$$
a b<\frac{\lambda_{1} \lambda_{2} \lambda_{3}^{2} \alpha^{r+s}}{(g-1)^{2}} \leq 16(g-1)^{2} \alpha^{r+s},
$$


and therefore

$$
a b<16(g-1)^{2} \alpha^{r+s}=16(g-1)^{2} \alpha^{r_{3}(1 / \delta+1 / \lambda)} \leq 16(g-1)^{2} \alpha^{5 r_{3} / 6} .
$$

On the other hand, we have

$$
(g-1) a b=\lambda_{3}\left(\alpha^{r_{3}}-\beta^{r_{3}}\right) \geq \alpha^{r_{3}}-\beta^{r_{3}} \geq \alpha^{r_{3}}-2(g-1),
$$

where we have used the fact that $\beta^{r_{3}}=\left(d_{3}+g-1\right) / \lambda_{3} \leq 2(g-1)$. Hence,

$$
\alpha^{r_{3}}-2(g-1) \leq(g-1) a b<16(g-1)^{3} \alpha^{5 r_{3} / 6},
$$

and a crude estimate now yields

$$
\alpha^{r_{3}}<16(g-1)^{3} \alpha^{5 r_{3} / 6}+2(g-1)<17(g-1)^{3} \alpha^{5 r_{3} / 6} .
$$

Thus,

$$
\alpha^{r_{3}}<17^{6}(g-1)^{18} .
$$

Now combining the various estimates we obtain

$$
\begin{aligned}
g^{n_{3}-1} & <\frac{d_{3}\left(g^{n_{3}}-1\right)}{g-1}-1=a b=\frac{\lambda_{3}}{g-1}\left(\alpha^{r_{3}}-\beta^{r_{3}}\right) \\
& <2 \alpha^{r_{3}}<2 \times 17^{6}(g-1)^{18} .
\end{aligned}
$$

Since $g \geq 3$, the above inequality gives $n_{3} \leq 28$, and therefore this case does not yield any solution with $n_{3} \geq 29$.

CASE 1.3: $r_{3}<\max \left\{r_{1}, r_{2}\right\}$. Assume for the moment that $r_{3}<r_{2}$. Then

$$
(g-1) a b=\lambda_{3}\left(\alpha^{r_{3}}-\beta^{r_{3}}\right) \text { and }(g-1) a c=\lambda_{2}\left(\alpha^{r_{2}}-\beta^{r_{2}}\right) .
$$

Write $\operatorname{gcd}\left(r_{2}, r_{3}\right)=r_{2} / \delta$ with some integer $\delta>1$. Then, as before,

$$
(g-1) a \leq \lambda_{2} \lambda_{3}\left(\alpha^{r_{2} / \delta}-\beta^{r_{2} / \delta}\right)
$$

and by the second equation of $(3.9)$, we get

$$
c \geq \frac{\lambda_{2}\left(\alpha^{r_{2}}-\beta^{r_{2}}\right)}{\lambda_{2} \lambda_{3}\left(\alpha^{r_{2} / \delta}-\beta^{r_{2} / \delta}\right)}>\frac{\alpha^{r_{2}(\delta-1) / \delta}}{2(g-1)} .
$$

The above bound yields

$$
2 \alpha^{r_{3}} \geq \frac{\lambda_{3}}{g-1}\left(\alpha^{r_{3}}-\beta^{r_{3}}\right)=a b>c^{2}>\frac{\alpha^{2 r_{2}(\delta-1) / \delta}}{4(g-1)^{2}} .
$$

If we assume that $\delta \geq 3$, then since $r_{2}>r_{3}$, we get

$$
2 r_{2}(\delta-1) / \delta>4 r_{3} / 3 \text {. }
$$

If we assume that $\delta=2$, then

$$
2 r_{2}(\delta-1) / \delta=r_{2}=2 r_{3}>4 r_{3} / 3 .
$$

In both cases inequality (3.11) implies

$$
\alpha^{r_{3} / 3}<8(g-1)^{2} .
$$


Hence,

$$
g^{n_{3}-1}<\frac{d_{3}\left(g^{n_{3}}-1\right)}{g-1}-1=a b=\frac{\lambda_{3}}{g-1}\left(\alpha^{r_{3}}-\beta^{r_{3}}\right)<2 \alpha^{r_{3}}<2^{10}(g-1)^{6},
$$

which has no solution for $n_{3} \geq 12$ and $g \geq 3$.

The case when $r_{1}>r_{3}$ can be dealt with similarly. In particular, instead of $(3.10)$ we obtain

$$
b \geq \frac{\alpha^{r_{1}(\delta-1) / \delta}}{2(g-1)},
$$

where $r_{1} / \delta=\operatorname{gcd}\left(r_{1}, r_{3}\right)$. Using the inequality $a b>b^{2}$ instead of $a b>c^{2}$ in the middle of (3.11), we obtain the same bound for $n_{3}$.

CASE 2: $x_{3} / y_{3}$ and $x_{2} / y_{2}$ are multiplicatively independent. By (3.1), we have

$$
\begin{aligned}
& (g-1) a b=d_{3} g^{n_{3}}-\left(d_{3}+g-1\right), \\
& (g-1) a c=d_{2} g^{n_{2}}-\left(d_{2}+g-1\right) .
\end{aligned}
$$

Hence, we get an upper bound for $a$ :

$$
(g-1) a \leq \operatorname{gcd}\left(d_{3} g^{n_{3}}-\left(d_{3}+g-1\right), d_{2} g^{n_{2}}-\left(d_{2}+g-1\right)\right) .
$$

Since, by assumption,

$$
\frac{x_{3}}{y_{3}}=\frac{d_{3} g^{n_{3}}}{d_{3}+g-1} \quad \text { and } \quad \frac{x_{2}}{y_{2}}=\frac{d_{2} g^{n_{2}}}{d_{2}+g-1}
$$

are multiplicatively independent, we may apply Lemma 2.1 with the parameters

$$
\left(t_{1}, w_{1}, t_{2}, w_{2}, k_{1}, k_{2}\right)=\left(d_{3}, d_{3}+g-1, d_{2}, d_{2}+g-1, n_{3}, n_{2}\right),
$$

where

$$
\max \left\{\left|t_{1}\right|,\left|w_{1}\right|,\left|t_{2}\right|,\left|w_{2}\right|\right\} \leq 2(g-1) \quad \text { and } \max \left\{k_{1}, k_{2}, 3\right\} \leq n_{3} .
$$

Thus, by Lemma 2.1 and 3.12 , for $a$ we get the upper bound

$$
a \leq 4(2 g-2)^{5 \sqrt{n_{3}}+1} \text {. }
$$

On the other hand, we have an upper bound for $n_{3}$ given by (3.3):

$$
n_{3}<\frac{2 \log a}{\log g}+1 \text {. }
$$

Combining (3.13) and (3.14), we obtain

$$
n_{3}<\frac{\left(10 \sqrt{n_{3}}+2\right) \log (2 g-2)+\log 16}{\log g}+1 .
$$

From 3.15, we get

$$
n_{3} \leq 178
$$


which actually occurs when $g=4$. Note that if $g=3$ then 3.15 yields $n_{3} \leq 171$, while for larger values of $g$ we obtain better upper bounds for $n_{3}$. In particular, we have $n_{3} \leq 105$ provided $g$ is large enough. If we only assume $g \geq 200$ and $g \geq 10^{6}$ we find that $n_{3} \leq 135$ and $n_{3} \leq 116$, respectively.

CASE $3: x_{3} / y_{3}$ and $x_{2} / y_{2}$ are multiplicatively dependent and $x_{3} / y_{3}$ and $x_{1} / y_{1}$ are not. As in Case 1 , we may write

$$
x_{3}=\alpha^{r_{3}}, y_{3}=\alpha^{r_{3}} \quad \text { and } \quad x_{2}=\alpha^{r_{2}}, y_{2}=\alpha^{r_{2}} .
$$

Note that in the proof of Case 1.3 we never used the quantity $r_{1}$ when we considered the case $r_{2}<r_{3}$. Therefore, we may assume $r_{3}>r_{2}$.

Similarly to Case 2, we find an upper bound for $b$, but we use

$$
\begin{aligned}
& (g-1) a b=d_{3} g^{n_{3}}-\left(d_{3}+g-1\right), \\
& (g-1) b c=d_{2} g^{n_{1}}-\left(d_{1}+g-1\right)
\end{aligned}
$$

instead. Therefore, by Lemma 2.1 , we obtain the upper bound

$$
b \leq 4(2 g-2)^{5 \sqrt{n_{3}}+1} .
$$

Next we want to find an upper bound for $a$. To this end, we consider

$$
a b=\frac{\lambda_{3}}{g-1}\left(\alpha^{r_{3}}-\beta^{r_{3}}\right) \quad \text { and } \quad a c=\frac{\lambda_{2}}{g-1}\left(\alpha^{r_{2}}-\beta^{r_{2}}\right) .
$$

Hence, we obtain

$$
(g-1) a \mid \lambda_{3} \lambda_{2} \operatorname{gcd}\left(\alpha^{r_{3}}-\beta^{r_{3}}, \alpha^{r_{2}}-\beta^{r_{2}}\right)<4(g-1)^{2} \alpha^{r},
$$

where $r=\operatorname{gcd}\left(r_{2}, r_{3}\right)$. Thus,

$$
a<4(g-1) \alpha^{r} .
$$

On the other hand,

$$
a b+1=\frac{d_{3}\left(g^{n_{3}}-1\right)}{g-1} \geq g^{n_{3}-1}+g^{n_{3}-2}+\cdots+1,
$$

that is, $a \geq g^{n_{3}-1} / b$, whence by 3.17 we get

$$
a \geq \frac{g^{n_{3}-1}}{4(2 g-2)^{5 \sqrt{n_{3}}+1}} .
$$

By using (3.17) and the fact that $d_{3} \geq 1$ and $d_{2} \leq g-1$, we find the following lower bound for $b$ :

$$
b \geq \frac{b}{c}=\frac{a b}{a c}>\frac{a b+1}{a c+1}=\frac{d_{3}\left(g^{n_{3}}-1\right)}{d_{2}\left(g^{n_{2}}-1\right)} \geq \frac{g^{n_{3}-n_{2}}}{g-1},
$$

which yields

$$
g^{n_{3}-n_{2}}<(g-1) b
$$

Recall that

$$
\alpha^{r_{2}}=\frac{d_{2} g^{n_{2}}}{\lambda_{2}} \geq \frac{g^{n_{2}}}{2(g-1)} \quad \text { and } \quad \alpha^{r_{3}}=\frac{d_{3} g^{n_{3}}}{\lambda_{3}} \leq(g-1) g^{n_{3}} .
$$


As in Case 1, let $r=\operatorname{gcd}\left(r_{2}, r_{3}\right)$. We then find that

$$
\alpha^{r} \leq \alpha^{r_{3}-r_{2}} \leq 2(g-1)^{2} g^{n_{3}-n_{2}}<2(g-1)^{3} b,
$$

where the last inequality is due to (3.21). We combine (3.17), (3.19), (3.20) and $(3.22)$ to obtain

$$
\begin{aligned}
\frac{g^{n_{3}-1}}{4(2 g-2)^{5 \sqrt{n_{3}}+1}} & \leq \frac{g^{n_{3}-1}}{b} \leq a<4(g-1) \alpha^{r} \\
& <8(g-1)^{4} b \leq 32(g-1)^{4}(2 g-2)^{5 \sqrt{n_{3}}+1} .
\end{aligned}
$$

Taking logarithms we obtain a similar inequality for $n_{3}$ to the one in Case 2:

$$
n_{3}<\frac{\left(10 \sqrt{n_{3}}+2\right) \log (2 g-2)+4 \log (g-1)+\log 128}{\log g}+1 .
$$

The above yields

$$
n_{3} \leq 186 .
$$

Note that we obtain $n_{3} \leq 186$ if $g \in\{4,5\}$, whereas in all other cases we obtain better bounds. In particular, if we assume that $g \geq 200$, then we obtain $n_{3} \leq 143$, and if we assume that $g \geq 10^{6}$, then we get $n_{3} \leq 124$. Finally, if $g$ is large enough, then we may even assume that $n_{3} \leq 113$.

Let us summarize our results so far:

Proposition 3.1. Assume equations (3.1) hold. Then $n_{3} \leq 186$. If we assume that $g \geq 200$ or that $g \geq 10^{6}$, then we have $n_{3} \leq 143$ and $n_{3} \leq 124$, respectively. Moreover, we may even assume that $n_{3} \leq 113$ if $g$ is large enough $\left(g>10^{153}\right)$.

Now a simple combinatorial argument concludes the proof of the first part of our theorem. Indeed, the distinct tuples $\left(n_{1}, d_{1}\right),\left(n_{2}, d_{2}\right),\left(n_{3}, d_{3}\right)$ may be selected from a set of cardinality $185(g-1)$ and altogether in

$$
(185 g-185)(185 g-186)(185 g-187)
$$

ways. Since only those results are acceptable where

$$
d_{1} \frac{g^{n_{1}}-1}{g-1}<d_{2} \frac{g^{n_{2}}-1}{g-1}<d_{3} \frac{g^{n_{3}}-1}{g-1},
$$

we are left with

$$
\frac{(185 g-185)(185 g-186)(185 g-187)}{6}
$$

possibilities for the tuple $\left(d_{1}, n_{1}, d_{2}, n_{2}, d_{3}, n_{3}\right)$. Further, for a given sextuple $\left(d_{1}, n_{1}, d_{2}, n_{2}, d_{3}, n_{3}\right)$, the system of equations (3.1) has at most one solution in positive integers $(a, b, c)$. Additionally, since $b \geq 2, d_{3} \leq g-1, n_{3} \leq 186$ and (3.1), the estimate for $a$ is trivial. This concludes the proof of the first part of Theorem 1.1 . 
4. Counting the number of triples. We are left with the proof of the last statement of Theorem 1.1. The main purpose of this section is to prove Theorem 4.1 below. Let $\tilde{\mathcal{R}}_{g}$ be the set of repdigits together with the integers of digit length 1 in base $g$. Denote by $\tilde{\mathcal{D}}_{g}$ the set of triples $(a, b, c) \in \mathbb{N}^{3}$ such that $1 \leq c<b<a$ and $a b+1, a c+1$ and $b c+1$ are elements of $\tilde{\mathcal{R}}_{g}$. We prove the following theorem:

THeOREM 4.1. We have

$$
\begin{array}{ll}
\# \tilde{\mathcal{D}}_{g} \asymp g^{3 / 2} & (g \rightarrow \infty), \\
\# \mathcal{D}_{g} \ll g^{1+o(1)} & (g \rightarrow \infty) .
\end{array}
$$

Since $g$ is fixed throughout this section, we will omit the index of $\mathcal{D}_{g}$ and $\tilde{\mathcal{D}}_{g}$ and write only $\mathcal{D}$ and $\tilde{\mathcal{D}}$, respectively. In the course of the proof of Theorem 4.1, we consider several subsets of $\tilde{\mathcal{D}}$ which will be denoted by $\mathcal{D}_{1}, \ldots, \mathcal{D}_{4}$. We emphasize that in the following a subscript of $\mathcal{D}$ does not refer to the base $g$, but instead to a certain subset of $\tilde{\mathcal{D}}$.

Proof. Clearly, $\tilde{\mathcal{D}}$ can also be identified with the set of all sextuples

$$
\left(d_{1}, d_{2}, d_{3}, n_{1}, n_{2}, n_{3}\right) \quad \text { where } \quad 1 \leq d_{i} \leq g-1 \quad \text { for } i=1,2,3,
$$

such that there exist positive integers $c<b<a$ satisfying (3.1). Under this identification and using Proposition 3.1, for $g$ large enough we have $n_{1} \leq n_{2} \leq n_{3} \leq 113$ and $1 \leq d_{i} \leq g-1$ for $i=1,2,3$. So, trivially, $\# \tilde{\mathcal{D}} \ll g^{3}$. Let us improve this trivial bound. Let $\mathcal{D}_{1}$ be the subset of $\tilde{\mathcal{D}}$ such that $n_{3}=1$, and $\mathcal{D}_{2}=\tilde{\mathcal{D}} \backslash \mathcal{D}_{1}$. We prove:

(i) $\# \mathcal{D}_{1} \asymp g^{3 / 2}$ as $g \rightarrow \infty$.

(ii) $\# \mathcal{D}_{2} \ll g^{1+o(1)}$ as $g \rightarrow \infty$.

The conclusion of Theorem 4.1 follows from (i), (ii) and the fact that

$$
\# \tilde{\mathcal{D}}=\# \mathcal{D}_{1}+\# \mathcal{D}_{2} \text {. }
$$

First, let us deal with (i). For the lower bound, we choose $a>b>c$ all three in $\{1, \ldots,\lfloor\sqrt{g-2}\rfloor\}$. For each of these choices,

$$
a b+1 \leq\lfloor\sqrt{g-2}\rfloor^{2}+1 \leq g-1,
$$

so $a b+1=d_{1} \in[1, g-1]$ and similarly $a c+1=d_{2}$ and $b c+1=d_{3}$. Thus, $(a, b, c)$ is in $\mathcal{D}_{1}$, and we get

$$
\# \mathcal{D}_{1} \geq\left(\begin{array}{c}
\lfloor\sqrt{g-2}\rfloor \\
3
\end{array}\right) \gg g^{3 / 2}
$$

For the upper bound, note that we have to count the integers $a>b>c$ 
satisfying (3.1) with $n_{1}=n_{2}=n_{3}=1$. In particular, we have to count the triples $(a, b, c)$ satisfying

$$
1 \leq a \leq g-2 \quad \text { and } \quad 1 \leq c<b<\min \left\{a, \frac{g-1}{a}\right\} .
$$

For fixed $a$ there are $\ll \min \{a, g / a\}^{2}$ pairs $(b, c)$ satisfying 4.2 . Therefore

$$
\# \mathcal{D}_{1} \ll \int_{1}^{g} \min \{a, g / a\}^{2} d a=\int_{1}^{\sqrt{g}} a^{2} d a+\int_{\sqrt{g}}^{g}\left(\frac{g}{a}\right)^{2} d a \ll g^{3 / 2},
$$

which is the desired upper bound.

For (ii), let $\mathcal{D}_{3}$ be the subset of $\mathcal{D}_{2}$ such that $n_{3} \geq 3$. Due to Proposition 3.1. for $g$ large enough we may assume that $n_{3} \leq 113$ and $d_{3} \leq g-1$. We look at

$$
a b=d_{3} \frac{g^{n_{3}}-1}{g-1}-1 .
$$

Clearly, since $n_{3} \geq 3$, we have $a^{2}>a b \geq\left(g^{2}+g+1\right)-1>g^{2}$, so $a>g$. Since $d_{3}$ and $g$ are fixed and $n_{3} \leq 113$, the number of ways of choosing $(a, b)$ such that $a>b$ and (4.3) holds is

$$
\tau\left(\frac{d_{3}\left(g^{n_{3}}-1\right)}{g-1}-1\right) \ll g^{o(1)} \quad \text { as } g \rightarrow \infty,
$$

where $\tau(n)$ is the number of divisors of $n$. The asymptotic bound on the right side follows from a well-known upper bound for the divisor function (e.g. see [18, Theorem 2.11] or [4, Chapter 7.4]). It remains to find out in how many ways we can choose $c$. Well, let us also fix $n_{2} \leq n_{3}$. Then $d_{2} \in\{1, \ldots, g-1\}$ is such that

$$
d_{2} \frac{g^{n_{2}}-1}{g-1} \equiv 1(\bmod a) .
$$

This puts $d_{2}$ into a fixed arithmetic progression $\alpha_{n_{2}}$ modulo $a$, where $\alpha_{n_{2}}$ is the inverse of $\left(g^{n_{2}}-1\right) /(g-1)$ modulo $a$. We show that this progression contains at most one value for $d_{2}$. Assuming this is not the case, let $d_{2}$ and $d_{2}^{\prime}$ be both congruent to $\alpha_{n_{2}}$ and in the interval $[1, g-1]$. Assume that $d_{2}<d_{2}^{\prime}$; then $a \mid d_{2}^{\prime}-d_{2}$, so

$$
g<a \leq d_{2}^{\prime}-d_{2} \leq g-2,
$$

which is false. This shows that indeed once $d_{3}, n_{3}$ and $a$ (hence also $b$ ) are determined, then any choice of $n_{2} \leq n_{3}$ determines $d_{2}$ (hence $c$ ) uniquely. Thus,

$$
\# \mathcal{D}_{3} \leq \sum_{d_{3}=1}^{g-1} \sum_{n_{3}=3}^{113} \sum_{n_{2}=1}^{n_{3}} \tau\left(\frac{d_{3}\left(g^{n_{3}}-1\right)}{g-1}-1\right) \ll g^{1+o(1)} \quad(g \rightarrow \infty) .
$$


It remains to find an upper bound for the cardinality of $\mathcal{D}_{4}:=\mathcal{D}_{2} \backslash \mathcal{D}_{3}$. These triples are the ones that have $n_{3}=2$. We fix $d_{3}$ and write

$$
a b=d_{3}(g+1)-1 \text {. }
$$

There are at most $\tau\left(d_{3}(g+1)-1\right)=g^{o(1)}$ possibilities for $a>b$ satisfying the above relation 4.5 as $g \rightarrow \infty$. It remains to determine the number of choices for $c$. Let us also fix $n_{2} \leq n_{3}=2$. Then determining $c$ is equivalent to determining the number of choices for $d_{2}$ such that

$$
d_{2} \frac{g^{n_{2}}-1}{g-1} \equiv 1(\bmod a), \quad 1 \leq d_{2} \leq g-1 .
$$

Congruence (4.6) puts $d_{2}$ in a certain fixed arithmetic progression modulo $a$, and the number of such numbers $1 \leq d_{2} \leq g-1$ is at most

$$
1+\left\lfloor\frac{g-1}{a}\right\rfloor .
$$

We assume that $a \leq g-1$, otherwise there is at most one choice for $d_{2}$, and the counting function of such examples is at most $g^{1+o(1)}$ by the argument for $\# \mathcal{D}_{3}$. Then the number of choices for $c$ is at most

$$
1+\left\lfloor\frac{g-1}{a}\right\rfloor \leq 1+\frac{g-1}{a}<\frac{2 g}{a} \leq \frac{2 g}{\sqrt{d_{3}(g+1)-1}} \ll \frac{\sqrt{g}}{\sqrt{d_{3}}} .
$$

This shows that

$$
\begin{aligned}
\# \mathcal{D}_{4} & \ll \sum_{n_{2} \leq 2} \sum_{d_{3}=1}^{g-1} \tau\left(d_{3}(g+1)-1\right) \frac{\sqrt{g}}{\sqrt{d_{3}}} \\
& \ll g^{1 / 2+o(1)} \sum_{1 \leq d_{3} \leq g-1} \frac{1}{\sqrt{d_{3}}} \ll g^{1 / 2+o(1)} \int_{1}^{g-1} \frac{d t}{t^{1 / 2}} \\
& \ll g^{1 / 2+o(1)}\left(\left.2 t^{1 / 2}\right|_{t=1} ^{t=g-1}\right) \ll g^{1+o(1)} \quad(g \rightarrow \infty) .
\end{aligned}
$$

Together with 4.4), we get

$$
\# \mathcal{D}_{2} \leq \# \mathcal{D}_{3}+\# \mathcal{D}_{4} \leq g^{1+o(1)} \quad(g \rightarrow \infty),
$$

which is (ii).

5. The case of small bases $g$. For the bases $2 \leq g \leq 200$ we have computed all triples $(a, b, c) \in \mathcal{D}_{g}$. In particular we found the following triples:

\begin{tabular}{cccc}
\hline$g$ & $a$ & $b$ & $c$ \\
\hline 23 & 65 & 17 & 7 \\
42 & 136 & 93 & 6 \\
104 & 292 & 187 & 32 \\
171 & 5607 & 619 & 5 \\
190 & 439 & 248 & 67 \\
\hline
\end{tabular}


In our computations, we considered all values of $2<g \leq 200$ one by one and we split our work depending on the size of $a$. If $g \leq 100$, we set $B:=1000$, and for $101 \leq g \leq 200$, we set $B:=10000$.

For every $a<B$ we proceed as follows: for $2 \leq b<a$ we check whether $a b+1$ is a repdigit number in base $g$. If yes, we also check if we can find $c<b$ such that $a b+1, a c+1$ and $b c+1$ are all repdigit numbers in base $g$.

For $a \geq B$ we proceed as follows: We use equations (3.1) and (3.2). For all integer values of $2 \leq n_{2} \leq 186$, and all integer values of $n_{3}$ between $n_{2}$, and the minimum of 186 and $2 n_{2}$, and for all possible digits $d_{2}$ and $d_{3}$, we compute

$$
a b=d_{3} \frac{g^{n_{3}}-1}{g-1}-1, \quad a c=d_{2} \frac{g^{n_{2}}-1}{g-1}-1 .
$$

Since $a \leq \operatorname{gcd}(a b, a c)$, the cases when $\operatorname{gcd}(a b, a c)<B$ are covered by the cases when $a<B$, so we only have further work to do if

$$
\operatorname{gcd}\left(d_{3} \frac{g^{n_{3}}-1}{g-1}-1, d_{2} \frac{g^{n_{2}}-1}{g-1}-1\right) \geq B
$$

In this case, for every integer $2 \leq n_{1} \leq n_{2}$ and every digit $d_{1}$, we check whether

$$
\left(d_{3} \frac{g^{n_{3}}-1}{g-1}-1\right)\left(d_{2} \frac{g^{n_{2}}-1}{g-1}-1\right)\left(d_{1} \frac{g^{n_{1}}-1}{g-1}-1\right)
$$

is a square, and if yes, then we check whether the corresponding values of $a, b$ and $c$ are integers. If yes, then we found a solution.

We implemented the above algorithm in Magma [2], and the running time was less than four days on an Intel(R) Core(TM) $9603.2 \mathrm{GHz}$ processor.

Acknowledgments. The research was supported in part by the University of Debrecen (A.B.), by the János Bolyai Scholarship of the Hungarian Academy of Sciences (A.B.), and by grants K100339 (A.B.) and NK104208 (A.B.) of the Hungarian National Foundation for Scientific Research. The research was also supported by the Austrian science found (FWF) under the project P 24801-N26.

\section{References}

[1] M. Alp, N. Irmak, and L. Szalay, Reduced diophantine quadruples with the binary recurrence $G_{n}=A G_{n-1}-G_{n-2}$, An. Ştiinţ. Univ. "Ovidius" Constanţa Ser. Mat. 23 (2015), 23-31.

[2] W. Bosma, J. Cannon, and C. Playoust, The Magma algebra system. I. The user language, J. Symbolic Comput. 24 (1997), 235-265.

[3] Y. Bugeaud and A. Dujella, On a problem of Diophantus for higher powers, Math. Proc. Cambridge Philos. Soc. 135 (2003), 1-10. 
[4] J.-M. De Koninck and F. Luca, Analytic Number Theory: Exploring the Anatomy of Integers, Grad. Stud. Math. 134, Amer. Math. Soc., Providence, RI, 2012.

[5] A. Dujella, Diophantine m-tuples, http://web.math.hr/ ${ }^{\sim}$ duje/dtuples.html.

[6] A. Dujella, There are only finitely many Diophantine quintuples, J. Reine Angew. Math. 566 (2004), 183-214.

[7] C. Fuchs, F. Luca, and L. Szalay, Diophantine triples with values in binary recurrences, Ann. Scuola Norm. Sup. Pisa Cl. Sci. (5) 7 (2008), 579-608.

[8] C. A. Gomez Ruiz and F. Luca, Tribonacci Diophantine quadruples, Glas. Mat. Ser. III 50 (2015), 17-24.

[9] C. A. Gomez Ruiz and F. Luca, Diophantine quadruples in the sequence of shifted Tribonacci numbers, Publ. Math. Debrecen 86 (2015), 473-491.

[10] N. Irmak and L. Szalay, Diophantine triples and reduced quadruples with the Lucas sequence of recurrence $u_{n}=A u_{n-1}-u_{n-2}$, Glas. Mat. Ser. III 49 (2014), 303-312.

[11] M. Keith, Repdigit polygonal numbers, J. Recreat. Math. 12 (1979/80), 9-15.

[12] T. Kovács, G. Péter, and N. Varga, On some polynomial values of repdigit numbers, Period. Math. Hungar. 67 (2013), 221-230.

[13] F. Luca and L. Szalay, Fibonacci Diophantine triples, Glas. Mat. Ser. III 43 (2008), 253-264.

[14] F. Luca and L. Szalay, Lucas Diophantine triples, Integers 9 (2009), \#A35, 441-457.

[15] F. Luca and V. Ziegler, Multiplicative relations on binary recurrences, Acta Arith. 161 (2013), 183-199.

[16] F. Luca and V. Ziegler, A note on the number of S-Diophantine quadruples, Comm. Math. 22 (2014), 49-55.

[17] D. Marques and A. Togbé, On repdigits as product of consecutive Fibonacci numbers, Rend. Istit. Mat. Univ. Trieste 44 (2012), 393-397.

[18] H. L. Montgomery and R. C. Vaughan, Multiplicative Number Theory. I. Classical Theory, Cambridge Stud. Adv. Math. 97, Cambridge Univ. Press, Cambridge, 2007.

[19] L. Szalay and V. Ziegler, On an S-unit variant of Diophantine m-tuples, Publ. Math. Debrecen 83 (2013), 97-121.

Attila Bérczes

Institute of Mathematics

University of Debrecen

P.O. Box 12

H-4010 Debrecen, Hungary

E-mail: berczesa@science.unideb.hu

István Pink

Institute of Mathematics

University of Debrecen

P.O. Box 12

H-4010 Debrecen, Hungary

and

University of Salzburg

Hellbrunnerstrasse 34/I

A-5020 Salzburg, Austria

E-mail: pinki@science.unideb.hu; istvan.pink@sbg.ac.at
Florian Luca

School of Mathematics

University of the Witwatersrand

Private Bag X3, Wits 2050

Johannesburg, South Africa

E-mail: florian.luca@wits.ac.za

Volker Ziegler

University of Salzburg

Hellbrunnerstrasse 34/I

A-5020 Salzburg, Austria

E-mail: volker.ziegler@sbg.ac.at 